\title{
Personality disorders and substance use disorders: a narrative review
}

Farhad Faridhosseini ${ }^{1}$, Ali Saghebi ${ }^{2}$, Mansoureh Mirzadeh ${ }^{3}$

${ }^{1}$ Associate Professor of Psychiatry, Psychiatry and Behavioral Sciences Research Center, Ibn-e-Sina Hospital, Mashhad University of Medical Sciences, Mashhad, Iran

${ }^{2}$ Assistant Professor of Psychiatry, Psychiatry and Behavioral Sciences Research Center, Ibn-e-Sina Hospital, Mashhad University of Medical Sciences, Mashhad, Iran

${ }^{3}$ Child and Adolescent Psychiatrist, Psychiatry and Behavioral Sciences Research Center, Ibn-e-Sina Hospital, Mashhad University of Medical Sciences, Mashhad, Iran

Type of article: Mini review

\begin{abstract}
Background: Personality factors have always been considered to play an important role in the etiology of substance-related disorders. Psychopathologic factors underlying an individual's personality structure have a major role in his or her tendency to use substances. This comorbidity can have an adverse effect on seeking and complying with treatment.

Objective: This review provides the comorbidity of personality disorders and substance use disorders (SUD).

Methods: In this report which was conducted in 2016, authors gathered data from different journals issued in print or electronic versions. These journals were indexed in Web of Science (Thomson Reuters), PubMed, Scopus, Google Scholar, ProQuest, Science Direct, Scientific Information Database (SID) and Magiran. A comprehensive search was done using keywords "Comorbidity", "Personality disorders" and "Substance use disorders". The research investigated in this review was conducted from January 1995 to 2016.

Results: Comorbidity of borderline personality disorder, antisocial personality and diagnostic and therapeutic considerations are the most well-known problems in personality disorder. In dealing with these patients special attention should be paid to their safety, suicidal and homicidal thoughts

Conclusion: It should be noted that an early diagnosis of comorbid personality disorder would lead to an adjusted expectancy from treatment, and adoption of long-term treatments for these patients

Keywords: Comorbidity, Personality disorders, Substance use disorders
\end{abstract}

\section{Introduction}

Comorbidity of personality with substance dependency is a global challenge and a huge concern for public health across the world. Personality factors have always been considered to play an important role in the etiology of substance-related disorders (1). According to early psychological theories, it was noted that people who tend to consume substances have impaired emotional regulation, and use these substances in order to control the constant and intense negative emotions. These people have difficulty controlling their impulses and seek pleasure in them. In recent theories, the role of defects in tolerance and management of painful emotions (such as anxiety, anger, or guilt) has been proposed. Unlike the old theory that focuses more on the role of intensity of negative emotions in the tendency to use substances, today, the inability to control and cope with such emotions are mostly emphasized. For instance, it is mentioned that opioids, alcohol, and nicotine are abused to control anger, anxiety, and depression, respectively. So it seems that the psychopathologic factors underlying an individual's personality structure have a major role in his or her tendency to use substances $(2,3)$. The high prevalence of psychiatric disorders in substance abusers has been shown in many studies. For instance, in the extensive study of the National Comorbidity Survey

\section{Corresponding author:}

Mansoureh Mirzadeh, Psychiatry and Behavioral Sciences Research Center, Ibn-e-Sina Hospital, Mashhad University of Medical Sciences, Mashhad, Iran.

Tel: +98.9155018705, Fax: +98.5137112722, E-mail: MirzadehM@mums.ac.ir

Received: November 06, 2016, Accepted: December 26, 2017, Published: June 2019

iThenticate screening: December 08, 2017, English editing: January 06, 2018, Quality control: November 26, 2018

(C) 2018 The Authors. This is an open access article under the terms of the Creative Commons Attribution-NonCommercialNoDerivs License, which permits use and distribution in any medium, provided the original work is properly cited, the use is non-commercial and no modifications or adaptations are made. 
(NCS), it was observed that $51 \%$ of substance-dependent patients have at least one other psychiatric diagnosis, including personality disorders. The percentage of comorbidity has been different in other studies (from $38 \%$ to $60 \%)(3,4)$. Antisocial personality disorder is the most common comorbid diagnosis in opioid and cocaine users $(5)$. The patients in Methadone Maintenance Treatment (MMT) programs in the Baltimore study, had 34.8\% lifetime comorbidity with personality disorders. The most common personality disorder was antisocial, and then came borderline and avoidant personality disorders (6). The comorbidity between Substance Use Disorders (SUDs) and cluster B personality disorders is more frequent than other personality disorders (7). Cluster B personality disorders present with impulsiveness, self-destructiveness, unstable emotions, and a disrupted pattern of relations (8-10). The comorbidity of these disorders and substance use can have an adverse effect on seeking and complying with treatment. In this article, the etiological factors of this comorbidity, especially concerning antisocial and borderline personality disorders, will be discussed.

\section{Material and Methods}

\subsection{Research design and search strategy}

Based on the objective of the present study which focuses on the comorbidity of personality disorders and substance use disorders that was conducted in 2016, various databases such as Web of Science (Thomson Reuters), PubMed, Scopus, Google Scholar, ProQuest, Science Direct, Scientific Information Database (SID) and Magiran were assessed. Keywords were: "Comorbidity,", "Personality disorders," and "Substance use disorders". The research investigated in this review was conducted from January 1995 to January 2016.

\subsection{Inclusion and exclusion criteria}

We included all cross-sectional, longitudinal, cohort or case-control analytical designs, descriptive studies and clinical trials reporting on comorbidity of personality disorders and substance use disorders, and published in peerreview journals. Only articles in English or Persian were included. Studies not related to this research were excluded.

\subsection{Quality assessment}

Researchers used all databases from 1970 to 2015, and by using keywords from 367 related documents extracted, 29 articles meeting the inclusion criteria were included. In this process, five steps were followed: 1 . identifying the research question; 2. search methods for identifying relevant studies; 3 . study selection; 4. charting the data, collating and summarizing; and 5. reporting the results.

\section{Results and discussion}

\subsection{Comorbidity of borderline personality disorder and SUD}

Borderline personality disorder (BPD) is a major health problem that has negative effects on individuals, families, and society. Affected individuals manifest problems in various areas including cognitions, emotions, behaviors, and interpersonal relationships $(11,12)$. Anger, self-mutilation, suicide, unstable emotions, and dissociative symptoms are common in these patients $(13,14)$. Surveys have displayed a high degree of comorbidity between BPD and SUD (3). More than $57.4 \%$ of patients with BPD are involved in some sort of SUD. On the other hand, 5\% to $32 \%$ of patients with SUD have BPD. Individuals with BPD begin substance abuse at an early age and have a worse prognosis in substance use trend. The adverse effects of substance abuse on the emotional, social, and legal aspects of their lives are more prominent. Moreover, treatment of comorbid BPD and SUD is more difficult than each one alone $(15,16)$. Impulsivity plays an essential role in the comorbidity of these disorders $(16,17)$. For an impulsive action, many dimensions can be considered, including unplanned action, involvement in destructive behaviors without considering the potential damages, impulsive aggression, and intolerance of delayed satisfaction of the demands and rewards $(18,19)$. The relationship between impulsivity as a personality trait that is common in patients with borderline personality disorder, and substance abuse, is unknown. It is also unclear whether it is the impulsivity trait that predisposes to substance abuse or dependence, or the consumption of substances that leads to impulsive behavior (20-22). Studies have shown that individuals with comorbid conditions have higher levels of impulsivity compared to each of the two disorders alone. Comparison of patients with SUD alone and patients with comorbid SUD and BPD reveals a higher level of behavioral control disorder in the latter with more frequent occurrence of destructive behaviors. Thus, it seems that the comorbidity of these two disorders has a synergistic effect on the psychopathologic aspects (21). Impulsivity can be studied from various aspects. One aspect is the discounting in the value attributed to rewards due to a delay in their presentation that is called delayed discounting procedure (DDP). In cocaine and heroin users the values of rewards decrease over time. This reduction is higher in substance users compared to the healthy group. This finding is also seen in people with BPD and it seems that these people have 
lower tolerance to a delayed satisfaction of their demands. Again, concerning comorbidity, the question is raised that which of the two disorders can lead to a disorder in the DDP. In Petry's study, it was found that compared with normal subjects, alcoholics had a more pronounced discounting of delayed rewards even when currently abstinent. As a result, it seems that the process of DDP is mostly that of a personality trait, and is not merely related to substance abuse; although the comorbidity of substance-related disorders and BPD intensifies it. The reason suggested for the comorbidity of these disorders is the existence of a common inherited predisposition to impulsivity. Decreased serotonin may be associated with impulsive aggression. This biological propensity, along with other biological and psychological characteristics, may be responsible for impulsive behavior in substancerelated disorders and borderline personality disorder. In addition, common environmental factors may contribute to the development of both disorders. Trait impulsivity can be exacerbated by environmental factors such as family conflict, parental abuse, or physical and emotional problems in children, factors that play a role in reducing brain serotonin. In addition, social learning plays a role in the intensification of impulsivity. In these individuals, substance abuse may be considered an adaptive psychological process that leads to the immediate benefit of reducing negative emotions despite their harmful outcomes besides DDP in the long run. Furthermore, the social environment has taught them that a delay in satisfying the demands can result in the fact that they may never reach them. Another factor that plays a role in the comorbidity of these disorders is the intolerance of negative affectivity, so that the earlier occurrence of each of the two disorders will lead to the occurrence of the other disorder, mediated by emotional instability and dominance of negative emotions and their intolerance. The existence of negative emotions in a BPD patient leads to a more destructive pattern of substance abuse and more craving for its use during therapy. Also, emotional instability and dominance of negative emotions caused by substance dependency leads to impulsivity, destructive behaviors, and intensification of borderline personality traits.

\subsection{Comorbidity of antisocial personality disorder and SUD}

Higher incidence of antisocial personality disorder and SUDs has been reported in many studies. Antisocial personality disorder has an early onset. These patients are impulsive and violent, and tend to have high risk-taking and cannot learn from their mistakes (23). Of patients with antisocial personality disorder, $80 \%$ have serious problems with alcohol or other substances during their lifetime. Comorbidity of antisocial personality disorder with substance abuse or dependence leads to a worse and more chronic course of both disorders, multiple substance use, more violence, premature discontinuation of treatment, and worse prognosis (24). Antisocial personality disorder, conduct disorder, and SUD are all known as externalizing disorders, the common characteristics of which are disinhibition, absence of sense of responsibility and conscience, limitlessness, increased novelty seeking, and sensation seeking (25-27). Impulsivity plays an essential role in the comorbidity of these disorders (28). In the fourfactor model of Whiteside \& Lynam, impulsivity is established from the four subscales of urgency, sensation seeking, lack of premeditation, and lack of perseverance (16). These are factors that lead to disinhibition and risktaking behaviors in different areas, including substance abuse. Patients with antisocial personality disorder and substance use related disorders have similar behaviors: they are often not aware of their problems or deny them, and if faced with the immediate rewards and future unfavorable consequences of their choices (including loss of job, family, etc.), they think only of the immediate reward (27). It has been observed that substance-dependent individuals with higher psychosocial functioning impairment score higher on the scales of psychopathy and antisocial behaviors. Therefore, it seems that a neurological disorder may be a common ground for both disorders. Concerning this kind of decision-making, i.e. choosing the rewards with immediate rewards but higher consequent losses, the role of ventromedial prefrontal cortex $(\operatorname{vmPFC})$ is mostly mentioned in the literature $(29,30)$.

\subsection{Diagnostic and therapeutic considerations}

Since the comorbidity of personality disorders and SUDs can lead to worse outcomes, an integration of the treatment of personality disorders into the therapeutic programs of substance-related abusers is very important. It should be noted that an early diagnosis of comorbid personality disorders would lead to an adjusted expectancy from treatment and adoption of long-term treatments for these patients. Moreover, by adopting appropriate strategies, their resistance to treatments can be mitigated (31). It is important to distinguish changes in personality and behavior resulting from intoxication with or withdrawal from substances from a comorbid personality disorder. The independent diagnosis of personality disorders is usually made when their symptoms have clearly existed before the onset of substance abuse and do not subside after a few consequent months of withdrawal. In the assessment and diagnosis of personality disorders by general practitioners (GPs), it is necessary to also take into account the family background (as a chaotic family environment increases the probability of diagnosing personality disorders), emotional and affective states, underlying personality, associated problems, patient's method of handling problems, and his or her capability of empathy. The most important challenge for the therapist in dealing with comorbid 
patients is establishing and maintaining a therapeutic relationship. A patient-centered approach should be adopted when interviewing and evaluating these patients, and the patient himself or herself should have active participation in the identification and finding a solution to his or her problems. Proper implementation of these techniques requires a strong therapeutic relationship with the patient. Regular planned meetings can contribute to strengthening the doctor-patient relationship and their positive interaction. In dealing with these patients, special attention should be paid to their safety, since destructive behaviors such as self-mutilation and suicidal and homicidal thoughts are common in some of them. In the treatment of these patients, both pharmacological and non-pharmacological therapies have significant roles. Pharmacotherapy is effective on symptoms such as affective dysregulation, cognitive distortions, and impaired impulse control; but it is better to seek help from psychotherapy in the treatment of interpersonal problems and character disorders. Therefore, a multimodal approach is needed to treat these comorbid individuals. Serotonin reuptake inhibitors, antipsychotics, and lithium are used in the treatment of aggression in patients with antisocial personality disorder. Serotonin reuptake inhibitors, such as fluoxetine, and anticonvulsant drugs, such as carbamazepine, are effective in the treatment of mood swings, impulsivity, and severe behavioral problems in people with BPD, and may improve their overall performance. Medications such as tricyclic antidepressants which can be lethal in overdose should be avoided because of the increased risk of suicide in people with BPD. It should be noted that there is a high risk of medication misuse of in these patients, and therefore, overprescription of medications, especially benzodiazepines, should be avoided. Benzodiazepines may lead to paradoxical disinhibition in these patients (32).

\section{Conclusions}

The most important challenge for the therapist in dealing with comorbid patients is establishing and maintaining a therapeutic relationship with them. A patient-centered approach should be adopted when interviewing and evaluating them, and the patient himself or herself should have active participation in identifying and finding a solution to his or her problems. In dealing with patients who have a comorbidity of personality disorders and SUDs, special attention should be paid to their safety since destructive behaviors, such as self-mutilation and suicidal and homicidal thoughts, are common in some of them.

\section{Acknowledgments:}

This study has not received any financial support for conducting this research. The authors would like to thank Psychiatry and Behavioral Sciences Research Center, Ibn-e-Sina Hospital, Mashhad University of Medical Sciences.

\section{Conflict of Interest:}

There is no conflict of interest to be declared.

\section{Authors' contributions:}

All authors contributed to this project and article equally. All authors read and approved the final manuscript.

\section{References:}

1) Cloninger CR, Svrakic DM. Personality disorders. The medical basis of psychiatry. Springer. 2016: 53750. doi: 10.1007/978-1-4939-2528-5_28.

2) Korsgaard HO, Torgersen S, Wentzel-Larsen T, Ulberg R. Substance abuse and personality disorder comorbidity in adolescent outpatients: are girls more severely ill than boys? Child Adolesc Psychiatry Ment Health. 2016; 10(1): 1. doi: 10.1186/s13034-016-0096-5. PMID: 27069507, PMCID: PMC4827187.

3) Carpenter RW, Wood PK, Trull TJ. Comorbidity of borderline personality disorder and lifetime substance use disorders in a nationally representative sample. J Pers Disord. 2016; 30(3): 336-50. doi: 10.1521/pedi_2015_29_197. PMID: 25893556.

4) Anthony JC, Warner LA, Kessler RC. Comparative epidemiology of dependence on tobacco, alcohol, controlled substances, and inhalants: basic findings from the National Comorbidity Survey. Exp Clin Psychopharm. 1994; 2(3): 244. doi: 10.1037//1064-1297.2.3.244.

5) Moeller FG, Dougherty DM, Barratt ES, Oderinde V, Mathias CW, Harper RA, et al. Increased impulsivity in cocaine dependent subjects independent of antisocial personality disorder and aggression. Drug Alcohol Depend. 2002; 68(1): 105-11. doi: 10.1016/S0376-8716(02)00106-0. PMID: 12167556.

6) Miller JD, Lyman DR, Widiger TA, Leukefeld C. Personality disorders as extreme variants of common personality dimensions: can the five factor model adequately represent psychopathy? J Pers. 2001; 69(2): 253-76. PMID: 11339798. 
7) Casillas A, Clark LA. Dependency, impulsivity and self-harm: traits hypothesized to underlie the association between cluster B personality and substance use disorders. J Pers Disord. 2002; 16(5): 424-36. PMID: 12489309.

8) Alemikhah M, Farid Hosseini f, Rajaei AR, Rasouli Azad M, Kordi H. Comparison of brain behavioral systems between methamphetamine dependent and normal people; based on Gray's revised reinforcement sensitivity theory. Journal of Fundamentals of Mental Health. 2013; 15(57): 17-26.

9) Ball SA, Tennen H, Poling JC, Kranzler HR, Rounsaville BJ. Personality, temperament, and character dimensions and the DSM-IV personality disorders in substance abusers. J Abnorm Psychol. 1997; 106(4): 545. doi: 10.1037/0021-843X.106.4.545. PMID: 9358685.

10) Rounsaville BJ, Kranzler HR, Ball S, Tennen H, Poling J, Triffleman E. Personality disorders in substance abusers: relation to substance use. J Nerv Ment Dis. 1998; 186(2): 87-95. doi: 10.1097/00005053199802000-00004. PMID: 9484308.

11) Tull MT, Gratz KL. The impact of borderline personality disorder on residential substance abuse treatment dropout among men. Drug Alcohol Depend. 2012; 121(1): 97-102. doi: 10.1016/j.drugalcdep.2011.08.014. PMID: 21907503, PMCID: PMC3237890.

12) Liotti G. Borderline Personality Disorders. Eur Psychiatry. 2015; 30: 4. doi: 10.1016/S09249338(15)30002-X.

13) Ritschel LA, Kilpela LS. Borderline Personality Disorder. The Encyclopedia of Clinical Psychology. 2015. doi: 10.1002/9781118625392.wbecp478.

14) Flory JD. Defining the construct of reactive aggression in borderline personality disorder: Commentary on "Aggression in borderline personality disorder-A multidimensional model". Personal Disord. 2015; 6(3): 294-5. doi: 10.1037/per0000134. PMID: 26191824.

15) Malayeri A, Kaviani H, Asadi S, Farid Hoseini F. Evaluation of personality dimensions using the Cloninger Temperament and Character Inventory in subjects with borderline personality disorders. Tehran Univ Med J. 2008; 66(9): 633-8.

16) Maraz A, Andó B, Rigó P, Harmatta J, Takách G, Zalka Z, et al. The two-faceted nature of impulsivity in patients with borderline personality disorder and substance use disorder. Drug Alcohol Depend. 2016; 163: 48-54. doi: 10.1016/j.drugalcdep.2016.03.015. PMID: 27107850.

17) Alemikhah M, Faridhosseini F, Kordi H, Rasouli-Azad M, Shahini N. Comparative Study of the Activity of Brain Behavioral Systems in Methamphetamine and Opiate Dependents. Int J High Risk Behav Addict. 2016; 5(1). doi: 10.5812/ijhrba.25075. PMID: 27218066, PMCID: PMC4870470.

18) Paris J. The nature of borderline personality disorder: multiple dimensions, multiple symptoms, but one category. J Pers Disord. 2007; 21(5): 457. doi: 10.1521/pedi.2007.21.5.457. PMID: 17953501.

19) Barker V, Romaniuk L, Cardinal R, Pope M, Nicol K, Hall J. Impulsivity in borderline personality disorder. Psychol Med. 2015; 45(09): 1955-64. doi: 10.1017/S0033291714003079. PMID: 25600066.

20) Bornovalova MA, Lejuez C, Daughters SB, Rosenthal MZ, Lynch TR. Impulsivity as a common process across borderline personality and substance use disorders. Clin Psychol Rev. 2005; 25(6): 790-812. doi: 10.1016/j.cpr.2005.05.005. PMID: 16005556.

21) Kruedelbach N, McCormick RA, Schulz SC, Grueneich R. Impulsivity, coping styles, and triggers for craving in substance abusers with borderline personality disorder. J Pers Disord. 1993; 7(3): 214-22. doi: 10.1521/pedi.1993.7.3.214.

22) Trull TJ, Sher KJ, Minks-Brown C, Durbin J, Burr R. Borderline personality disorder and substance use disorders: A review and integration. Clin Psychol Rev. 2000; 20(2): 235-53. doi: 10.1016/S02727358(99)00028-8. PMID: 10721499.

23) Derefinko KJ, Widiger TA. Antisocial personality disorder. The medical basis of psychiatry: Springer. 2016: 229-45. doi: 10.1007/978-1-4939-2528-5_13.

24) Li D, Zhao H, Kranzler HR, Oslin D, Anton RF, Farrer LA, et al. Association of COL25A1 with comorbid antisocial personality disorder and substance dependence. Biol Psychiatry. 2012; 71(8): 733-40. doi: 10.1016/j.biopsych.2011.12.011. PMID: 22297151, PMCID: PMC3548659.

25) Farid Hoseini F, Kaviani H, Asadi SM, Ali Malayeri N, Moghaddas Bayat MR. Character and temperament in patients with anti-social personality and its comparison with Iran society norm. Cognitive Sciences News 2004; 9: 54-60.

26) Moody L, Franck C, Bickel WK. Comorbid depression, antisocial personality, and substance dependence: Relationship with delay discounting. Drug Alcohol Depend. 2016; 160: 190-6. doi: 10.1016/j.drugalcdep.2016.01.009. PMID: 26846198, PMCID: PMC4767536. 
27) Repo-Tiihonen E, Hallikainen T. Antisocial personality disorder. Duodecim. 2015; 132(2): 130-6. PMID: 26939485.

28) Alcorn JL 3rd, Gowin JL, Green CE, Swann AC, Moeller FG, Lane SD. Aggression, impulsivity, and psychopathic traits in combined antisocial personality disorder and substance use disorder. J Neuropsychiatry Clin Neurosci. 2013; 25(3): 229-32. doi: 10.1176/appi.neuropsych.12030060. PMID: 24026715, PMCID: PMC4777890.

29) Farid Hosseini f. Evaluation of risky decision-making in patients with antisocial personality disorders and its relationship with personality dimensions. 2007.

30) Bechara A, Damasio H. Decision-making and addiction (part I): impaired activation of somatic states in substance dependent individuals when pondering decisions with negative future consequences. Neuropsychologia. 2002; 40(10): 1675-89. PMID: 11992656.

31) Devens M. Personality disorders. Primary Care: Clinics in Office Practice. 2007; 34(3): 623-40. doi: 10.1016/j.pop.2007.05.008.

32) Kraus G, Reynolds DJ. The "AB-C's" of the Cluster B's: Identifying, understanding, and treating Cluster B personality disorders. Clin Psychol Rev. 2001; 21(3): 345-73. doi: 10.1016/S0272-7358(99)00052-5. PMID: 11288605 . 